COMMUNICATIONS IN

ANALYSIS AND GEOMETRY

Volume 13, Number 2, 461-478, 2005

\title{
Characterization for Balls by Potential Function of Kähler-Einstein Metrics for Domains in $\mathbb{C}^{n}$
}

\author{
SONG-YING LI
}

\section{Introduction and Main results.}

Let $D$ be a bounded domain in $\mathbb{C}^{n}$. For $u \in C^{2}(D)$, we denote $H(u)(z)=$ $\left[\frac{\partial^{2} u}{\partial z_{i} \partial \bar{z}_{j}}\right]_{n \times n}$ the complex hessian matrix of $u$ at $z$. A plurisubharmonic function $U(z)$ on $D$ is called a potential function for the Kähler-Einstein metric if $U$ satisfies the Monge-Ampère equation:

$$
\operatorname{det} H(U)=e^{(n+1) U} \quad \text { in } D ; \quad \text { and } \quad U=\infty \text { on } \partial D \text {. }
$$

Equivalently the function

$$
\rho(z)=-e^{-U(z)}, \quad z \in D
$$

is called the potential function for the Fefferman's metric, and $\rho$ satisfies the Fefferman equation:

$$
J(\rho)=-\operatorname{det}\left[\begin{array}{cc}
\rho & \bar{\partial} \rho \\
(\bar{\partial} \rho)^{*} & H(\rho)
\end{array}\right]=1, \quad z \in D, \quad \rho(z)=0 \quad \text { on } \partial D,
$$

where $\bar{\partial} \rho$ denotes the row vector with entries $\frac{\partial \rho}{\partial \bar{z}_{1}}, \cdots, \frac{\partial \rho}{\partial \bar{z}_{n}}$. When $D$ is a smoothly bounded strictly pseudoconvex domain in $\mathbb{C}^{n}$, a formal positive solution of (1.3) was given by C. Fefferman in [7]. The existence of a positive solution was proved by Cheng and Yau [5]. Moreover, they also proved that $\rho \in C^{n+3 / 2}(\bar{D})$. Lee and Melrose [21] gave an asymptotic expansion for $\rho$, which implies that $\rho \in C^{n+2-\epsilon}(\bar{D})$. When $D$ is a smoothly bounded weakly pseudoconvex domain in $\mathbb{C}^{n}$, it was proved by Cheng and Yau [5] that there is a complete Kähler-Einstein metric. The same result on the existence of a complete Kähler-Einstein metric was obtained later by Mok and Yau in [27] without an assumption on the smoothness of the boundary $\partial D$.

Several very interesting and fundamental theorems on the characterization of the unit ball in $\mathbb{C}^{n}$ have bee discovered before. For example, B. 
Wong's characterization theorem for the unit ball by using non-compact automorphism group in [29] or [17]. A celebrated theorem of Stoll in [28] and Burns in [3] on a characterization theorem of the ball, by using the degenerate complex Monge-Ampère equation, can be stated as follows:

Theorem 1.1. Let $M$ be a complex manifold of dimension $n$. If there is a smooth strictly plurisubharmoinc function $u: M \rightarrow[0,1)$ so that it is onto and satisfies

$$
\operatorname{det} H(\log u)(z)=0, \quad z \in\{z \in M: u(z)>0\},
$$

then there is a biholomorphic map $\phi: M \rightarrow B_{n}$.

For convenience, we let

$$
\tilde{U}(z)=U(z)-c_{0} \quad \text { with } c_{0}=\min \{U(z): z \in D\} .
$$

Then $U$ satisfies (1.1) if and only if $\tilde{U}$ satisfies

$\operatorname{det} H(U)=g(z) e^{(n+1) U}$ in $D, \quad U=\infty \quad$ on $\partial D, \quad \min \{U(z): z \in D\}=0$ with $g(z) \equiv e^{(n+1) c_{0}}$. In other words, $\tilde{\rho}(z)=\rho(z) e^{c_{0}}=-e^{-\left(U-c_{0}\right)}=-e^{-\tilde{U}}$ satisfies

$$
J(\rho)=g(z) \text { in } D, \quad \rho=0 \text { on } \partial D \quad \min \{\rho(z): z \in D\}=-1
$$

with $g(z) \equiv e^{(n+1) c_{0}}$.

One observes that if $\phi: D \rightarrow B_{n}$ is a biholomorphic map with $\phi\left(z_{0}\right)=0$, then

$$
U(z)=-\log \left(1-|\phi(z)|^{2}\right)
$$

satisfies (1.6) with

$$
g(z)=\left|\operatorname{det} \phi^{\prime}(z)\right|^{2}>0
$$

and $\log g(z)$ being pluriharmonic in $D$. Moreover, if $\rho(z)=-e^{-U(z)}$ then

$$
\operatorname{det} H(\log (1+\rho(z)))=\operatorname{det} H\left(\log |\phi|^{2}\right)=0, \quad z \in D \backslash\left\{z_{0}\right\} .
$$

Conversely, we shall prove the following main theorem of this paper. 
Theorem 1.2. Let $D$ be a smoothly bounded weakly pseudoconvex domain in $\mathbb{C}^{n}$. Let $U$ be pluri-subharmonic in $D$ such that (1.6) holds for some positive function $g$ in $D$ with $\log g(z)$ being bounded and pluriharmonic in D. Then

(a) If $\rho(z)=-e^{-U(z)}$ satisfies that

$$
\liminf _{z \rightarrow \partial D} \operatorname{det} H(\log (1+\rho(z))) \geq 0,
$$

then $D$ is biholomorphically equivalent to the unit ball in $\mathbb{C}^{n}$.

(b) In addition to the assumption (1.11), if $g(z) \equiv c$ for some positive constant $c$, then there is a biholomorphic map $\phi: D \rightarrow B_{n}$ so that $\operatorname{det} \phi^{\prime}(z)=$ $\sqrt{c}$ on $D$.

The paper is organized as follows: In Section 2, we will provide several fundamental results, which are mainly stated in Theorem 2.1. As a corollary of Theorem 2.1, we prove Part (a) of Theorem 1.2. In Section 3, we first prove a theorem building up a relationship between subharmonicity of $\log \left(1-e^{-U}\right)$ in $D$ and the biholomorphic mapping from $D$ to $B_{n}$ with constant Jacobian. As an application, we will prove Part (b) of Theorem 1.2.

\section{The Proof of Part (a) Theorem 1.2.}

In this section, we will prove several preliminary results related to the KählerEinstein metric. We will also give a few characterizations of the unit ball using some quantities associated to the Kähler-Einstein metric. Mainly, we will prove the following theorem.

Theorem 2.1. Let $D$ be a bounded weakly pseudoconvex domain in $\mathbb{C}^{n}$. Let $U$ be a strictly plurisubharmonic solution of the Monge-Ampère equation:

$\operatorname{det} H(U)=g(z) e^{(n+1) U}$ in $D ; u=\infty$ on $\partial D$ and $\min \{U(z): z \in D\}=0$,

where $g(z)>0$ and $\log g(z)$ is pluriharmonic in D. Let $\rho(z)=-e^{-U(z)}$. Then the following three statements hold.

(a) If $\log (1+\rho(z))$ is plurisubharmonic in $D$ then $D$ is biholomorphically equivalent to the unit ball $B_{n}$ in $\mathbb{C}^{n}$.

(b) If $\lim _{z \rightarrow w} e^{U(z)}\left(U^{i \bar{j}} U_{i} U_{\bar{j}}+e^{-U(z)}-1\right)=0$ for all $w \in \partial D$ then $D$ is biholomorphically equivalent to $B_{n}$. 
(c) If $\liminf _{z \rightarrow \xi}\left(g(z)^{-1} \operatorname{det}(H(\rho)(z)) \geq 1\right.$ for all $\xi \in \partial D$, then $D$ is biholomorphically equivalent to $B_{n}$.

Notice that without the condition $m=\min \{U(z): z \in D\}=0$, a theorem of Cheng and Yau in [5] shows that (2.1) has a strictly plurisubhamonic solution $U(z) \in C^{\infty}(D)$ so that, the Kähler-Einstein metric $U_{i \bar{j}} d z_{i} d \bar{z}_{j}$ is complete on $D$. Therefore $U-m$ is a strictly plurisubharmonic solution of (2.1) if we replace $g(z)$ by $g(z) e^{(n+1) m}$.

Let

$$
U_{i}=\frac{\partial U}{\partial z_{i}}, \quad U_{\bar{j}}=\frac{\partial U}{\partial \bar{z}_{j}} \quad \text { and } \quad U_{i \bar{j}}=\frac{\partial^{2} U}{\partial z_{i} \partial \bar{z}_{j}} .
$$

and let $H(U)=\left[U_{i \bar{j}}(z)\right]_{n \times n}$ be positive definite on $D$. We use the notation $\left[U^{i \bar{j}}\right]_{n \times n}=\left(H(U)^{-1}\right)^{t}$ and

$$
|\partial U|^{2}(z)=U^{i \bar{j}} U_{i} U_{\bar{j}}(z)
$$

Let

$$
\rho(z)=-e^{-U(z)}, \quad v(z)=1+\rho(z), \quad u(z)=\log v(z) .
$$

We will prove the following lemma.

Lemma 2.2. Let $U(z)$ be strictly pluri-subharmonic and satisfy (2.1). Then

$$
\operatorname{det} H(u)(z)=\frac{g(z)}{v(z)^{n+1}} e^{U(z)}\left(1-e^{-U(z)}-|\partial U|^{2}\right)
$$

and

$$
\operatorname{det} H(v)(z)=g(z) e^{U(z)}\left(1-|\partial U|^{2}\right) .
$$

Proof. Since

$$
\partial_{i} u(z)=\frac{1}{v(z)} \partial_{i} v(z)=\frac{e^{-U(z)} U_{i}(z)}{v(z)}
$$

and

$$
\begin{aligned}
\partial_{i \bar{j}} u(z) & =\partial_{\bar{j}}\left[\frac{e^{-U(z)} U_{i}(z)}{v(z)}\right] \\
& =\frac{e^{-U(z)}}{v(z)}\left[-U_{i} U_{\bar{j}}+U_{i \bar{j}}-\frac{e^{-U} U_{i} U_{\bar{j}}}{v}\right] \\
& =\frac{e^{-U(z)}}{v(z)}\left[U_{i \bar{j}}-\frac{e^{-U}+v(z)}{v} U_{i} U_{\bar{j}}\right] \\
& =\frac{e^{-U(z)}}{v(z)}\left[U_{i \bar{j}}-\frac{1}{v} U_{i} U_{\bar{j}}\right]
\end{aligned}
$$


Let $\partial U$ denote the row vector with entries $\partial_{1} U, \cdots, \partial_{n} U$. Then

$$
\begin{aligned}
\operatorname{det} H(u)(z) & =\frac{e^{-n U(z)}}{v(z)^{n}} \operatorname{det}\left(H(U)(z)-\frac{1}{v(z)}(\partial U)^{*}(\partial U)\right) \\
& =\frac{e^{-n U(z)}}{v(z)^{n}} \operatorname{det}(H(U)(z))\left(1-\frac{1}{v(z)}(\partial U) H(U)^{-1}(\partial U)^{*}\right) \\
& =\frac{e^{-n U(z)}}{v(z)^{n}} g(z) e^{(n+1) U}\left(1-\frac{1}{v(z)}(\partial U) H(U)^{-1}(\partial U)^{*}\right) \\
& =g(z) \frac{e^{U(z)}}{v(z)^{n}}\left(1-\frac{1}{v(z)}|\partial U|^{2}\right) \\
& =g(z) \frac{e^{U(z)}}{v(z)^{n+1}}\left(1-e^{-U(z)}-|\partial U|^{2}\right),
\end{aligned}
$$

and

$$
\begin{aligned}
\operatorname{det}\left(v_{i \bar{j}}\right)(z) & =\operatorname{det}\left(e^{-U(z)}\left[U_{i \bar{j}}-U_{i} U_{\bar{j}}\right]\right) \\
& =e^{-n U(z)} \operatorname{det}\left(U_{i \bar{j}}\right)\left(1-|\partial U(z)|^{2}\right) \\
& =g(z) e^{U(z)}\left(1-|\partial U(z)|^{2}\right) .
\end{aligned}
$$

Therefore, (2.5) and (2.6) hold, and the proof of the lemma is complete. $\quad$

Corollary 2.3. Let $U(z)$ be a strictly plurisubharmonic solution of (2.1). Let $u(z)$ and $v(z)$ be defined by (2.4) in term of $U$. Then the following statements hold.

(a) $u(z)$ is plurisubharmonic in $D$ if and only if $1-e^{-U(z)}-|\partial U|^{2}(z) \geq 0$ on $D$.

(b) If $u(z)$ is plurisubharmonic in $D$ then $v(z)$ is strictly plurisubharmonic in $D$.

Proof. We first prove Part (a). Since $U$ is strictly plurisubharmonic in $D$ and

$$
u_{i \bar{j}}(z)=\frac{e^{-U(z)}}{v(z)}\left(U_{i \bar{j}}-\frac{1}{v(z)} U_{i} U_{\bar{j}}\right)
$$

one can easily see that $u(z)$ is plurisubharmonic in $D$ if and only if $\operatorname{det}\left(u_{i \bar{j}}\right) \geq$ 0 on $D$. The Part (a) follows from (2.5).

Next we prove Part (b). Since $u$ is plurisubharmonic in $D$ then $|\partial U|^{2}+e^{-U(z)} \leq 1$ by Part (a). Thus $v(z)=e^{u(z)}$ is plurisubharmonic in $D$. Moreover,

$$
\operatorname{det}\left(v_{i \bar{j}}\right)=g(z) e^{U}\left(1-|\partial U|^{2}\right) \geq g(z) e^{U} e^{-U(z)}=g(z)>0 .
$$


Therefore, $v$ is strictly plurisubharmonic in $D$; and the proof of the corollary is complete.

For simplicity, we shall use the notation:

$$
T(z)=U^{i \bar{j}} U_{i} U_{\bar{j}}+e^{-U(z)}=|\partial U|^{2}+e^{-U(z)} .
$$

In order to prove Theorem 2.1, We will need the following theorem.

Theorem 2.4. Let $D$ be a bounded weakly pseudoconvex domain in $\mathbb{C}^{n}$. Let $U$ be a strictly plurisubharmonic solution of (2.1). Then

(a) If $T(z) \leq 1$ on $D$ then $T(z) \equiv 1$ on $D$.

(b) If $\lim \sup _{z \rightarrow \xi} e^{U(z)}(T(z)-1)=0$ for any $\xi \in \partial D$ then $T(z) \equiv 1$ on $D$.

Proof. Let

$$
\mathcal{L}=U^{k \bar{\ell}} \partial_{k \bar{\ell}}+2 \operatorname{Re}\left(U^{k \bar{\ell}} U_{k} \partial_{\bar{\ell}}\right) .
$$

Then if $T(z) \leq 1$ in $D$ then we shall prove

$$
\mathcal{L} T(z) \geq 0 \quad \text { on } \quad D .
$$

For any $w \in D$, we shall calculate $\mathcal{L} T$ at $w$. Let $z=\phi(\xi)$ be a biholomorphic map between a neighborhood of 0 and a neighborhood of $w$. Then we define

$$
\tilde{U}(\xi)=U(\phi(\xi)) .
$$

It is easy to choose such a map $\phi$ so that $\phi(0)=w$ and, in the new coordinates,

$$
\tilde{U}_{i \bar{j} k}(0)=0, \quad 1 \leq i, j, k \leq n .
$$

By the definition of $T(z)$, if we let

$$
V(z)=U^{i \bar{j}} U_{i} U_{\bar{j}}
$$

and

$$
\tilde{V}(\xi)=\tilde{U}^{i \bar{j}} \tilde{U}_{i} \tilde{U}_{\bar{j}}(\xi), \quad \tilde{T}(\xi)=\tilde{V}(\xi)+e^{-\tilde{U}(\xi)},
$$

then

$$
\tilde{T}(\xi)=T(\phi(\xi))=\tilde{V}(\xi)+e^{-\tilde{U}(\xi)}
$$


and

$$
\operatorname{det}\left(\tilde{U}_{i \bar{j}}\right)(\xi)=|f(\xi)|^{2} g(\phi(\xi)) e^{(n+1) \tilde{U}(\xi)}=F(\xi) e^{(n+1) \tilde{U}(\xi)}
$$

where $f(\xi)=\operatorname{det}\left(\phi^{\prime}(\xi)\right)$ and $F(\xi)=|f(\xi)|^{2} g(\phi(\xi))$. Thus

$\tilde{V}_{k}(\xi)=\partial_{k}\left[\tilde{U}^{i \bar{j}}\right] \tilde{U}_{i} \tilde{U}_{\bar{j}}+\tilde{U}^{i \bar{j}} \tilde{U}_{i k} \tilde{U}_{\bar{j}}+\tilde{U}^{i \bar{j}} \tilde{U}_{i} \tilde{U}_{k \bar{j}}=\partial_{k}\left[\tilde{U}^{i \bar{j}}\right] \tilde{U}_{i} \tilde{U}_{\bar{j}}+\tilde{U}^{i \bar{j}} \tilde{U}_{i k} \tilde{U}_{\bar{j}}+\tilde{U}_{k}$.

Since $\tilde{U}_{i \bar{j} k}(0)=0$ and

$$
\frac{\partial \tilde{U}^{i \bar{j}}}{\partial \xi_{k}}=\frac{\partial \tilde{U}^{i \bar{j}}}{\partial \tilde{U}_{p \bar{q}}} \frac{\partial \tilde{U}_{p \bar{q}}}{\partial \xi_{k}}=-\tilde{U}^{p \bar{j}} \tilde{U}^{i \bar{q}} \tilde{U}_{p \bar{q} k}
$$

we have

$$
\frac{\partial \tilde{U}^{i \bar{j}}}{\partial \xi_{k}}(0)=\frac{\partial \tilde{U}^{i \bar{j}}}{\partial \bar{\xi}_{k}}(0)=0 .
$$

and

$$
\frac{\partial^{2} \tilde{U}^{i \bar{j}}}{\partial \xi_{k} \partial \bar{\xi}_{\ell}}(0)=-\sum_{p, q=1}^{n} \tilde{U}^{p \bar{j}} \tilde{U}^{i \bar{q}}(0) \tilde{U}_{p \bar{q} k \bar{\ell}}(0) .
$$

Therefore, at $\xi=0$, we have

$$
\begin{aligned}
\tilde{V}_{k \bar{\ell}}(0)= & \partial_{k \bar{\ell}}\left[\tilde{U}^{i j}\right] \tilde{U}_{i} \tilde{U}_{\bar{j}}+\partial_{k}\left[\tilde{U}^{i \bar{j}}\right] \tilde{U}_{i \bar{\ell}} \tilde{U}_{\bar{j}}+\partial_{k}\left[\tilde{U}^{i \bar{j}}\right] \tilde{U}_{i} \tilde{U}_{\bar{j} \ell} \\
& +\partial_{\bar{\ell}}\left[\tilde{U}^{i \bar{j}}\right] \tilde{U}_{i k} \tilde{U}_{\bar{j}}+\tilde{U}^{i \bar{j}} \tilde{U}_{i k \bar{\ell}} \tilde{U}_{\bar{j}}+\tilde{U}^{i \bar{j}} \tilde{U}_{i k} \tilde{U}_{\overline{j \ell}}+\tilde{U}_{k \bar{\ell}} \\
= & \partial_{k \bar{\ell}}\left[\tilde{U}^{i \bar{j}}\right] \tilde{U}_{i} \tilde{U}_{\bar{j}}+\tilde{U}^{i \bar{j}} \tilde{U}_{i k} \tilde{U}_{\overline{j \ell}}+\tilde{U}_{k \bar{\ell}} \\
= & -\sum_{p, q=1}^{n} \tilde{U}^{p \bar{j}} \tilde{U}^{i \bar{q}} \tilde{U}_{p \bar{q} k \bar{\ell}} \tilde{U}_{i} \tilde{U}_{\bar{j}}+\tilde{U}^{i \bar{j}} \tilde{U}_{i k} \tilde{U}_{\overline{j \ell}}+\tilde{U}_{k \bar{\ell}} .
\end{aligned}
$$

Since $f$ is holomorphic, $f(0) \neq 0$ and $\log g(\phi(\xi))$ is pluriharmonic, we have $\partial_{p \bar{q}} \log F=\partial_{p \bar{q}}\left(\log |f|^{2}+\log g(\phi(\xi))\right)=0$ near $\xi=0$. By (2.11) and (2.15), we have

$$
\sum_{k, \ell=1}^{n} \tilde{U}^{k \bar{\ell}} \partial_{k \bar{\ell}} \tilde{U}_{p \bar{q}}(0)=\partial_{p \bar{q}}[(n+1) \tilde{U}+\log F](0)=(n+1) \tilde{U}_{p \bar{q}}(0) .
$$

Therefore, at $\xi=0$ we have

$$
\tilde{U}^{k \bar{\ell}} \tilde{V}_{k \bar{\ell}}(0)=-(n+1) \tilde{U}^{p \bar{j}} \tilde{U}^{i \bar{q}} \tilde{U}_{p \bar{q}}(0) \tilde{U}_{i} \tilde{U}_{\bar{j}}+\tilde{U}^{k \bar{\ell}} \tilde{U}^{i \bar{j}} \tilde{U}_{i k} \tilde{U}_{\overline{j \ell}}(0)+n
$$




$$
\begin{aligned}
& =-(n+1) \tilde{U}^{i \bar{j}}(0) \tilde{U}_{i} \tilde{U}_{\bar{j}}+\tilde{U}^{k \bar{\ell}} \tilde{U}^{i \bar{j}} \tilde{U}_{i k} \tilde{U}_{\overline{j \ell}}(0)+n \\
& =-(n+1) \tilde{V}(0)+\tilde{U}^{k \bar{\ell}} \tilde{U}^{i \bar{j}} \tilde{U}_{i k} \tilde{U}_{\bar{j} \bar{l}}(0)+n
\end{aligned}
$$

and

$$
\tilde{U}^{k \bar{\ell}} \partial_{k \bar{\ell}}\left(e^{-\tilde{U}(\xi)}\right)=e^{-\tilde{U}(\xi)}\left(-\tilde{U}^{k \bar{\ell}} \tilde{U}_{k \bar{\ell}}+\tilde{U}^{k \bar{\ell}} \tilde{U}_{k} \tilde{U}_{\bar{\ell}}\right)=e^{-\tilde{U}(\xi)}(-n+\tilde{V}(\xi)) .
$$

Therefore, by combining (2.22) and (2.23)

$$
\begin{aligned}
& \tilde{U}^{k \bar{\ell}} \partial_{k \bar{\ell}} \tilde{T}(0) \\
& =-(n+1) \tilde{V}(0)+n+e^{-\tilde{U}(0)}[-n+\tilde{V}(0)]+\tilde{U}^{i \bar{j}} \tilde{U}^{k \bar{\ell}} \tilde{U}_{i k} \tilde{U}_{\overline{j \ell}} \\
& =n-n \tilde{V}(0)-n e^{-\tilde{U}(0)}-\tilde{V}(0)+\tilde{V}(0) e^{-\tilde{U}(0)}+\tilde{U}^{i \bar{j}} \tilde{U}^{k \bar{\ell}} \tilde{U}_{i k} \tilde{U}_{\overline{j \ell}} \\
& =n[1-\tilde{T}(0)]-\tilde{V}(0)\left[1-e^{-\tilde{U}(0)}\right]+\tilde{U}^{i \bar{j}} \tilde{U}^{k \bar{\ell}} \tilde{U}_{i k} \tilde{U}_{\bar{j} \bar{\ell}} .
\end{aligned}
$$

Now we let

$$
\tilde{\mathcal{L}}=\tilde{U}^{k \bar{\ell}} \frac{\partial^{2}}{\partial \xi_{k} \partial \bar{\xi}_{\ell}}+2 \operatorname{Re}\left(\tilde{U}^{k \bar{\ell}} \tilde{U}_{k} \frac{\partial}{\partial \bar{\xi}_{\ell}}\right)
$$

Then

$$
(\mathcal{L} h)(\phi(\xi))=\tilde{\mathcal{L}} \tilde{h}(\xi)
$$

and $\tilde{h}(\xi)=h(\phi(\xi))$ for any $h \in C^{2}(D)$.

Since $\tilde{U}_{i \bar{j} k}(0)=0$, we have $\partial_{i} \tilde{U}^{k \bar{\ell}}(0)=0$ and $\partial_{\bar{j}} \tilde{U}^{k \bar{\ell}}(0)=0$. Thus

$$
\begin{aligned}
\partial_{i} \tilde{T}(0) & =\partial_{i}\left(\tilde{U}^{k \bar{\ell}} \tilde{U}_{k} \tilde{U}_{\bar{\ell}}+e^{-\tilde{U}}\right)(0) \\
& =0+\tilde{U}^{k \bar{\ell}} \tilde{U}_{i k} \tilde{U}_{\bar{\ell}}(0)+\tilde{U}^{k \bar{\ell}} \tilde{U}_{k} \tilde{U}_{i \bar{\ell}}(0)-e^{-\tilde{U}(0)} \tilde{U}_{i}(0) \\
& =\tilde{U}^{k \bar{\ell}} \tilde{U}_{i k} \tilde{U}_{\bar{\ell}}(0)+\tilde{U}_{i}\left(1-e^{-\tilde{U}(0)}\right)
\end{aligned}
$$

and

$$
\partial_{\bar{j}} \tilde{T}(0)=\tilde{U}^{k \bar{\ell}} \tilde{U}_{k} \tilde{U}_{\overline{j \ell}}(0)+\tilde{U}_{\bar{j}}(0)\left(1-e^{-\tilde{U}(0)}\right) .
$$

Thus

$$
\tilde{U}^{i \bar{j}} \partial_{i} \tilde{T}(0) \tilde{U}_{\bar{j}}(0)=\tilde{U}^{i \bar{j}} \tilde{U}^{k \bar{\ell}} \tilde{U}_{\bar{j}} \tilde{U}_{i k} \tilde{U}_{\bar{\ell}}(0)+\tilde{V}(0)\left(1-e^{-\tilde{U}(0)}\right),
$$

and

$$
\tilde{U}^{i \bar{j}} \partial_{\bar{j}} \tilde{T}(0) \tilde{U}_{i}(0)=\tilde{U}^{i \bar{j}} \tilde{U}^{k \bar{\ell}} \tilde{U}_{i} \tilde{U}_{k} \tilde{U}_{\overline{j \ell}}(0)+\tilde{V}(0)\left(1-e^{-\tilde{U}(0)}\right) .
$$


Therefore

$$
\begin{aligned}
\operatorname{Re}\left(\tilde{U}^{k \bar{\ell}} \tilde{U}_{k} \partial_{\bar{\ell}}\right) \tilde{T}(0)= & \operatorname{Re}\left(\tilde{U}^{k \bar{\ell}} \tilde{U}_{k} \tilde{U}^{i \bar{j}} \tilde{U}_{i} \tilde{U}_{\overline{j \ell}}\right)+\tilde{V}(0)\left(1-e^{-\tilde{U}(0)}\right) \\
\geq & -\left(\sum_{i, j, k, \ell=1}^{n} \tilde{U}^{i \bar{j}} \tilde{U}^{k \bar{U}^{2}} \tilde{U}_{i k} \tilde{U}_{\overline{j \ell}}\right)^{1 / 2}\left(\sum_{i, j=1}^{n} \tilde{U}^{i \bar{j}} \tilde{U}_{i} \tilde{U}_{\bar{j}}\right) \\
& +\tilde{V}(0)\left(1-e^{-\tilde{U}(0)}\right) \\
= & -\left(\sum_{i, j, k, \ell=1}^{n} \tilde{U}^{i \bar{j}} \tilde{U}^{k \bar{\ell}^{\prime}} \tilde{U}_{i k} \tilde{U}_{\overline{j \ell}}\right)^{1 / 2} \tilde{V}+\tilde{V}(0)\left(1-e^{-\tilde{U}(0)}\right) .
\end{aligned}
$$

Since $\tilde{T}(0)=\tilde{V}(0)+e^{-\tilde{U}(0)} \leq 1$, we have $\tilde{V}(0)\left(1-e^{-\tilde{U}(0)}\right) \geq \tilde{V}(0)^{2}$. Thus

$$
\begin{aligned}
\mathcal{L} T(w)= & \tilde{\mathcal{L}} \tilde{T}(0) \\
\geq & n[1-\tilde{T}(0)]-2\left(\sum_{i, j, k, \ell=1}^{n} \tilde{U}^{i \bar{j}} \tilde{U}^{k \bar{\ell}} \tilde{U}_{i k} \tilde{U}_{\overline{j \ell}}\right)^{1 / 2} \tilde{V}(0) \\
& +\tilde{V}(0)\left(1-e^{-\tilde{U}(0)}\right)+\tilde{U}^{i \bar{j}} \tilde{U}^{k \bar{\ell}} \tilde{U}_{i k} \tilde{U}_{\overline{j \ell}}(0) \\
\geq & n[1-\tilde{T}(0)]-2\left(\sum_{i, j, k, \ell=1}^{n} \tilde{U}^{i \bar{j}} \tilde{U}^{k \bar{\ell}} \tilde{U}_{i k} \tilde{U}_{\overline{j \ell}}\right)^{1 / 2} \tilde{V}(0)+\tilde{V}(0)^{2} \\
& +\tilde{U}^{i \bar{j}} \tilde{U}^{k \bar{\ell}} \tilde{U}_{i k} \tilde{U}_{\bar{j} \bar{\ell}}(0) \\
= & n[1-\tilde{T}(0)]+\left[\tilde{V}(0)-\left(\sum_{i, j, k, \ell=1}^{n} \tilde{U}^{i \bar{j}} \tilde{U}^{k{ }_{\ell}} \tilde{U}_{i k} \tilde{U}_{\overline{j \ell}}\right)^{1 / 2}\right]^{2} \\
\geq & 0 .
\end{aligned}
$$

Therefore, since $w \in D$ is arbitrary, $\mathcal{L} T(z) \geq 0$ on $D$. Let $z_{0} \in D$ so that $U\left(z_{0}\right)=\min \{U(z): z \in D\}=0$. Then $T\left(z_{0}\right)=1$. Since $T(z) \leq 1$ and $T\left(z_{0}\right)=1$. By the maximum principle, we have $T(z) \equiv T\left(z_{0}\right)=1$, and the proof of Part (a) is complete.

Next we prove Part (b). Using (2.24), (2.29) and (2.30), we have

$$
\begin{aligned}
e^{-U(w)} U^{i \bar{j}} \partial_{i \bar{j}}\left[e^{U(z)}(T(z)-1)\right](w) \\
\quad=e^{-\tilde{U}(0)} \tilde{U}^{i \bar{j}} \partial_{i \bar{j}}\left[e^{\tilde{U}(\xi)}(\tilde{T}(\xi)-1)\right](0) \\
\quad=\tilde{U}^{i \bar{j}}\left(\tilde{U}_{i \bar{j}}+\tilde{U}_{i} \tilde{U}_{\bar{j}}\right)(\tilde{T}(0)-1)+\tilde{U}^{i \bar{j}}\left(\partial_{i} \tilde{T}(0) \tilde{U}_{\bar{j}}+\tilde{U}_{i} \partial_{\bar{j}} \tilde{T}(0)\right)+\tilde{U}^{i \bar{j}} \partial_{i \bar{j}} \tilde{T}(0) \\
=(n+\tilde{V}(0))(\tilde{T}(0)-1)+\tilde{U}^{i \bar{j}}\left(\partial_{i} \tilde{T}(0) \tilde{U}_{\bar{j}}+\tilde{U}_{i} \partial_{\bar{j}} \tilde{T}(0)\right)+\tilde{U}^{i \bar{j}} \partial_{i \bar{j}} \tilde{T}(0)
\end{aligned}
$$




$$
\begin{aligned}
= & (n+\tilde{V}(0))(\tilde{T}(0)-1)+\tilde{U}^{i \bar{j}} \tilde{U}^{k \bar{\ell}}\left(\tilde{U}_{\bar{j}} \tilde{U}_{i k} \tilde{U}_{\bar{\ell}}(0)+\tilde{U}_{i} \tilde{U}_{k} \tilde{U}_{\overline{j \ell}}(0)\right) \\
& +2 \tilde{V}(0)\left(1-e^{-\tilde{U}(0)}\right)+n[1-\tilde{T}(0)]-\tilde{V}(0)\left[1-e^{-\tilde{U}(0)}\right]+\tilde{U}^{i \bar{j}} \tilde{U}^{k \bar{\ell}} \tilde{U}_{i k} \tilde{U}_{\bar{j} \bar{\ell}} \\
= & \tilde{V}(0)(\tilde{T}(0)-1)+\tilde{U}^{i \bar{j}} \tilde{U}^{k \bar{\ell}}\left(\tilde{U}_{\bar{j}} \tilde{U}_{i k} \tilde{U}_{\bar{\ell}}(0)+\tilde{U}_{i} \tilde{U}_{k} \tilde{U}_{\overline{j \ell}}(0)\right) \\
& +\tilde{V}(0)[1-\tilde{T}(0)]+\tilde{V}(0)^{2}+\tilde{U}^{i \bar{j}} \tilde{U}^{k \bar{\ell}} \tilde{U}_{i k} \tilde{U}_{\overline{j \ell}} \\
= & \tilde{U}^{i \bar{j}} \tilde{U}^{k \bar{\ell}}\left(\tilde{U}_{\bar{j}} \tilde{U}_{i k} \tilde{U}_{\bar{\ell}}(0)+\tilde{U}_{i} \tilde{U}_{k} \tilde{U}_{\bar{j}(}(0)\right)+\tilde{V}(0)^{2}+\tilde{U}^{i \bar{j}} \tilde{U}^{k \bar{\ell}} \tilde{U}_{i k} \tilde{U}_{\bar{j} \bar{l}} \\
\geq & 0 .
\end{aligned}
$$

Therefore $e^{U(z)}(T(z)-1)$ attains its maximum over $\bar{D}$ at some point on $\partial D$. By the assumption: $\limsup _{z \rightarrow \xi} e^{U(z)}(T(z)-1)=0$ for all $\xi \in \partial D$, we have that $e^{U(z)}(T(z)-1) \leq 0$ on $D$. This implies that $T(z) \leq 1$ on $D$. By Part (a), we have that $T(z) \equiv 1$ on $D$, and the proof of Part (b) is complete. Therefore, we have completed the proof of Theorem 2.4. $\quad$ Q

From the proof of Theorem 2.4, one has the following corollary.

Corollary 2.5. Let $D$ be a smoothly bounded strictly pseudoconvex domain in $\mathbb{C}^{n}$. Let $U$ be a plurisubharmonic solution of (2.1) with $g \in C^{2}(\bar{D})$ being positive and $\log g$ being pluriharmonic in $D$. Let $\rho(z)=-e^{-U(z)}$. Then the function

$$
\frac{1}{g(z)} \operatorname{det} H(\rho)(z)
$$

attains its minimum over $\bar{D}$ at some point in $\partial D$.

Proof. By the results in [5], we have $\rho \in C^{2}(\bar{D})$. By (2.6) and definition of $v(z)$, one can easily see that

$$
\frac{1}{g(z)} \operatorname{det} H\left(-e^{-U}\right)(z)=e^{U(z)}\left(1-|\partial U|^{2}\right)=e^{U(z)}(1-T(z))+1, \quad z \in D
$$

The proof of Part (b) of Theorem 2.4 shows that $e^{U(z)}(T(z)-1)$ attains its maximum over $\bar{D}$ at some point in $\partial D$. This implies that the statement of Corollary 2.5 holds.

Now we are ready to prove Theorem 2.1.

\section{The proof of Theorem 2.1.}

Proof of Part (a) of Theorem 2.1: Since $u(z)=\log \left(1-e^{-U(z)}\right)$ is plurisubharmonic, we have by $(2.5)$ that $1-T(z) \geq 0$ on $D$. By Part (a) of Theorem 2.4, we have $T(z) \equiv 1$. By $(2.5)$ again, we have $\operatorname{det} H(u)(z)=$ 0 on $\{z \in D: u(z)>-\infty\}$. By Corollary 2.3,v(z)=e $e^{u(z)}$ is strictly 
plurisubharmonic in $D$ which maps $D$ onto $[0,1)$. Theorem 1.1 (of Burns and Stoll) implies that $D$ is biholomorphic to the unit ball in $\mathbb{C}^{n}$. This proves Part (a).

Proof of Part (b) of Theorem 2.1: The assumption of Part (b) of Theorem 2.1 is the same as the assumption of Part (b) of Theorem 2.4. Hence, Part (b) of Theorem 2.4 implies that $T(z) \equiv 1$ on $D$. Therefore, The proofs of Part (b) of Theorem 2.1 follows from Corollary 2.3 and Theorem 1.1 (of Burns and Stoll).

Proof of Part (c) of Theorem 2.1: By (2.6), we have

$$
\operatorname{det} H(v)(z)=g(z) e^{U(z)}(1-V(z))=g(z) e^{U(z)}(1-T(z))+g(z) .
$$

Thus

$$
\operatorname{det} H\left(-e^{-U}\right)(z)-g(z)=g(z) e^{U(z)}(1-T(z))
$$

By the assumption (c) of Theorem 2.1, we have

$$
\begin{aligned}
\liminf _{z \rightarrow \partial D} e^{U(z)}(1-T(z)) & =\liminf _{z \rightarrow \partial D}\left(\frac{\operatorname{det} H\left(-e^{-U}\right)(z)}{g(z)}-1\right) \\
& \geq 1-1=0 .
\end{aligned}
$$

Since $e^{U(z)}(1-T(z))$ attains its minimum over $\bar{D}$ at some point on $\partial D$, we have $e^{U(z)}(1-T(z)) \geq 0$ on $D$ and so $T(z) \leq 1$. By Theorem 2.4 (a), we have $T(z) \equiv 1$. Corollary 2.3 implies that the function

$$
v(z)=1-e^{-U(z)}, \quad z \in D
$$

is strictly plurisubharmonic in $D$, and $v: D \rightarrow[0,1)$ is onto. Moreover, $\operatorname{det} H(\log v(z))=0$ on $\{z \in D: v(z)>0\}$. Applying Theorem 1.1 (of Burns and Stoll), we have that $D$ is biholomorphically equivalent to the unit ball in $\mathbb{C}^{n}$.

\section{The proof of Part (b) of Theorem 1.2.}

In order to prove Part (b) of Theorem 1.2, we first consider biholomorphic mapping between $D$ and $B_{n}$.

Let $\phi: D \rightarrow B_{n}$ be a biholomorphic mapping. Let

$$
U(z)=-\log \left(1-|\phi(z)|^{2}\right)+\frac{1}{n+1} \log \left|\operatorname{det} \phi^{\prime}(z)\right|^{2}-c,
$$


with

$$
c=\min \left\{-\log \left(1-|\phi|^{2}\right)+\frac{1}{n+1} \log \left|\phi^{\prime}(z)\right|^{2}: z \in D\right\} .
$$

Since $U(z)$ is plurisubharmonic, there is point $z_{0} \in D$ so that $U\left(z_{0}\right)=0$. Without loss of generality (since $B_{n}$ is a symmetric domain), we may assume that

$$
\phi\left(z_{0}\right)=0
$$

Then $U$ is a strictly plurisubharmonic solution of $(2.1)$ with $g(z)=e^{(n+1) c}$. In particular, $\min \{U(z): z \in D\}=0$.

Let $\psi: B_{n} \rightarrow D$ be the inverse mapping of $\phi(z)$. Then

$$
\operatorname{det} \phi^{\prime}(\psi(w))=\frac{1}{\operatorname{det} \psi^{\prime}(w)}, \quad w \in B_{n}
$$

and

$$
\tilde{U}(w)=U(\psi(w))=-\log \left(1-|w|^{2}\right)-\frac{1}{n+1} \log \left|\operatorname{det} \psi^{\prime}(w)\right|^{2}-c .
$$

We shall prove the following theorem.

Theorem 3.1. Let $\phi: D \rightarrow B_{n}$ be a biholomorphic mapping so that $\phi\left(z_{0}\right)=0$. Let $U(z)$ be defined by (3.1) and (3.2). If $u(z)=\log \left(1-e^{-U(z)}\right)$ is plurisubharmonic near $\partial D$, then $\operatorname{det} \phi^{\prime}(z)=$ constant. In particular,

(i) If $n=1$ then $D$ is a disk;

(ii) If $D$ is circular with respect to a pint $w \in D$, then $\phi$ is a linear map: $\phi(z)=A(z-w)$, where $A$ is an $n \times n$ non-singular scalar matrix.

Proof. Let $\psi(w)=\phi^{-1}(w): B_{n} \rightarrow D$, and let

$\tilde{U}(w)=U(\psi(w))=-\log \left(1-|w|^{2}\right)-\log h(w), h(w)=\left|\left(\operatorname{det} \psi^{\prime}(w)\right)^{1 /(n+1)}\right|^{2} e^{c}$.

Then $h(0)=1$. Let

$$
\tilde{v}(w)=1-\left(1-|w|^{2}\right) h(w), \quad \text { and } \quad \tilde{u}(w)=\log \tilde{v}(w) .
$$

Then by (2.5)

$$
\operatorname{det} H(\tilde{u})(w)=\frac{h(w)^{n+1}}{\tilde{v}(w)^{n+1}} e^{\tilde{U}}\left(1-\left(1-|w|^{2}\right) h(w)-|\partial \tilde{U}|^{2}\right)
$$


where

$$
|\partial \tilde{U}|^{2}=\tilde{U}^{i} \tilde{U}_{i} \tilde{U}_{\bar{j}}, \quad \partial_{i} \tilde{U}=\frac{\bar{w}_{i}}{1-|w|^{2}}-\partial_{i} \log h(w)
$$

and

$$
\tilde{U}_{i \bar{j}}=\frac{\delta_{i j}}{1-|w|^{2}}+\frac{\bar{w}_{i} w_{j}}{\left(1-|w|^{2}\right)^{2}}, \quad \tilde{U}^{i \bar{j}}=\left(1-|w|^{2}\right)\left(\delta_{i j}-w_{i} \bar{w}_{j}\right) .
$$

Let

$$
X_{j}=\frac{\partial}{\partial w_{j}}-\bar{w}_{j} R, \quad R=\sum_{k=1}^{n} w_{j} \frac{\partial}{\partial w_{j}} .
$$

Then

$$
\begin{aligned}
|\partial \tilde{U}|^{2}= & \left(1-|w|^{2}\right)\left(\delta_{i j}-w_{i} \bar{w}_{j}\right)\left(\frac{\bar{w}_{i}}{1-|w|^{2}}-\partial_{i} \log h(w)\right)\left(\frac{w_{j}}{1-|w|^{2}}-\partial_{j} \log h(w)\right) \\
= & |w|^{2}-\left(\delta_{i j}-w_{i} \bar{w}_{j}\right)\left[\bar{w}_{i} \partial_{\bar{j}} \log h(w)+w_{j} \partial_{i} \log h(w)\right] \\
& +\left(1-|w|^{2}\right)\left(\delta_{i j}-w_{i} \bar{w}_{j}\right) \partial_{i} \log h(w) \partial_{\bar{j}} \log h(w) \\
= & |w|^{2}-\left(1-|w|^{2}\right)(R+\bar{R}) \log h(w) \\
& +\left(1-|w|^{2}\right)\left(\delta_{i j}-w_{i} \bar{w}_{j}\right) \partial_{i} \log h(w) \partial_{\bar{j}} \log h(w)
\end{aligned}
$$

Thus

$$
1-|\partial \tilde{U}|^{2}=\left(1-|w|^{2}\right)\left[1+(R+\bar{R}) \log h(w)-\left(\delta_{i j}-w_{i} \bar{w}_{j}\right) \partial_{i} \log h(w) \partial_{j} \log h(w)\right] .
$$

Therefore,

$$
\begin{aligned}
& h(w) e^{\tilde{U}}\left(1-|\partial \tilde{U}|^{2}-e^{-\tilde{U}}\right) \\
& =\left[1+(R+\bar{R}) \log h(w)-\left(\delta_{i j}-w_{i} \bar{w}_{j}\right) \partial_{i} \log h(w) \partial_{j} \log h(w)-h(w)\right] \\
& =\left[1+(R+\bar{R}) \log h(w)-\sum_{j=1}^{n}\left|X_{j} \log h\right|^{2}-\left(1-|w|^{2}\right)|R \log h(w)|^{2}-h(w)\right] .
\end{aligned}
$$

Since $\log \left(1-e^{-U(z)}\right)$ is plurisubharmonic in $D$, so is $\log \left(1-e^{-\tilde{U}}\right)$ in $B_{n}$. By (3.8) we have

$$
\frac{h(w)^{n+1}}{\tilde{v}(w)^{n+1}} e^{\tilde{U}(w)}\left(1-|\partial \tilde{U}(w)|^{2}-e^{-\tilde{U}(z)}\right) \geq 0, \quad w \in B_{n}
$$

Since $h>0$ and $v>0$ (if $w \neq 0$ ), we have

$$
h(w) e^{\tilde{U}(w)}\left(1-|\partial \tilde{U}(w)|^{2}-e^{-\tilde{U}(z)}\right) \geq 0, \quad w \in B_{n} .
$$


Moreover, since $h(w)$ is positive subharmonic and $\log h(w)$ is pluriharmonic, we have

$$
\begin{aligned}
0 & \leq \frac{1}{\sigma\left(\partial B_{n}\right)} \int_{\partial B_{n}} h(w) e^{\tilde{U}}\left(1-|\partial \tilde{U}|^{2}-e^{-\tilde{U}}\right) d \sigma(w) \\
& =\frac{1}{\sigma\left(\partial B_{n}\right)} \int_{\partial B_{n}}\left[1+(R+\bar{R}) \log h(w)-\sum_{j=1}^{n}\left|X_{j} \log h\right|^{2}-h(w)\right] d \sigma(w) \\
& =1-\frac{1}{\sigma\left(\partial B_{n}\right)} \int_{\partial B_{n}}\left[\sum_{j=1}^{n}\left|X_{j} \log h\right|^{2}+h(w)\right] d \sigma(w) \\
& \leq 1-h(0)-\frac{1}{\sigma\left(\partial B_{n}\right)} \int_{\partial B_{n}} \sum_{j=1}^{n}\left|X_{j} \log h\right|^{2} d \sigma(w) \\
& =-\frac{1}{\sigma\left(\partial B_{n}\right)} \int_{\partial B_{n}} \sum_{j=1}^{n}\left|X_{j} \log h\right|^{2} d \sigma(w) \\
& \leq 0
\end{aligned}
$$

This implies that

$$
\sum_{j=1}^{n}\left|X_{j} \log h\right| \equiv 0, \quad \text { on } \quad \partial B_{n}
$$

and

$$
\int_{\partial B_{n}} h(w) d \sigma(w)=h(0) \sigma\left(\partial B_{n}\right)=\sigma\left(\partial B_{n}\right)
$$

Since $\log h(z)$ is real-valued, (3.9) implies that $\log h(z)$ is CR. It must be a constant on $\partial B_{n}$. Since $\log h(w)$ is pluriharmonic, by the Maximum and Minimum Principles, we have $h(w) \equiv h(0)=1$ on $B_{n}$. This implies that $\left|\operatorname{det} \psi^{\prime}(w)\right|^{2}=e^{-(n+1) c}$. Since $\operatorname{det} \psi^{\prime}(w)$ is holomorphic, we have

$$
\operatorname{det} \psi^{\prime}(w) \equiv e^{i \theta} e^{-(n+1) c / 2}
$$

for some $\theta \in[0,2 \pi)$.

Parts (i) and (ii) follow directly from the Cartan's theorem (see [17]). Therefore, the proof of Theorem 3.1 is complete. $\quad$ ]

As a corollary of Theorem 2.1 and Theorem 3.1, we have

Corollary 3.2. Let $D$ be a bounded weakly pseudoconvex domain in $\mathbb{C}^{n}$ with $C^{2}$ boundary. Let $U(z)$ be a plurisubharmonic solution of (2.1) in $D$ with $g(z)=e^{(n+1) c}$ for some constant $c$ so that $\exp (-U) \in C^{2}(\bar{D})$ and

$$
\log (1-\exp (-U(z)))
$$


is plurisubharmonic near $\partial D$. Then there is a biholomorphic map $\phi: D \rightarrow$ $B_{n}$ so that $\operatorname{det} \phi^{\prime}(z)=e^{i \theta} e^{(n+1) c / 2}$ for some $\theta \in[0,2 \pi)$. In particular, when $n=1$ or $D$ is a circular domain with respect to $w \in D$, then $D$ must be the unit ball after a linear transformation.

Proof. By Theorem 2.1, we have that $D$ is biholomorphic to the unit ball $B_{n}$ in $\mathbb{C}^{n}$. Let $\phi: D \rightarrow B_{n}$ be a biholomorphic map. Let

$$
U^{0}(z)=-\log \left(1-|\phi|^{2}\right)+\frac{1}{n+1} \log \left|\operatorname{det} \phi^{\prime}(z)\right|^{2}-c, \quad z \in D .
$$

It is easy to show that

$$
\operatorname{det} H\left(U^{0}\right)(z)=e^{(n+1) c} e^{(n+1) U^{0}}, \quad \min \left\{U^{0}(z): z \in D\right\}=0
$$

Since $e^{-U} \in C^{2}(\bar{D})$, there exist two constants $0<c<C<\infty$ such that

$$
c H\left(U^{0}\right) \leq H(U) \leq C H\left(U^{0}\right), \quad z \in D .
$$

By the uniqueness theorem in [5], we have that $U(z)=U^{0}(z)$. Thus $\min \left\{U^{0}(z): z \in D\right\}=0$. Applying Theorem 3.1, we have that $\operatorname{det}\left(\phi^{\prime}\right) \equiv$ $e^{i \theta} e^{(n+1) c / 2}$ for some $\theta \in[0,2 \pi)$. Moreover, if $D$ is circular with respect to $w \in D$, Cartan's Theorem implies that $\phi(z)=A(z-w)$ with $A$ being a scalar $n \times n$ matric. Therefore, the proof of the corollary is complete.

Now we are ready to prove Part (b) of Theorem 1.2.

Proof. By Part (a) of Theorem 1.2, we have that $D$ is biholomorphic to the unit ball in $\mathbb{C}^{n}$. Corollary 3.2 implies that there is a biholomorphic map $\phi: D \rightarrow B_{n}$ so that $\operatorname{det} \phi^{\prime}(z) \equiv$ constant in $D$. This completes the proof of Part (b) of Theorem 1.2. $\quad$ व

Finally in the section, we connect the domains, which are biholomorphically equivalent to the unit ball with constant Jacobian maps, to the Bergman kernel function $K(z, w)$.

Proposition 3.3. Let $D$ be a bounded domain in $\mathbb{C}^{n}$ which is biholomorphically equivalent to a ball in $\mathbb{C}^{n}$. Then there is $a \in D$ such that $K(z, a)$ is constant function of $z$ if and only if there is a biholomorphic map $\phi: D \rightarrow B_{n}$ with $\operatorname{det} \phi^{\prime}(z)=$ constant.

Proof. If $\phi: D \rightarrow B_{n}$ is a biholomorphic map with $\operatorname{det} \phi^{\prime}(z)=$ constant $=c$, then

$$
K_{D}(z, w)=(1-\langle\phi(z), \phi(w)\rangle)^{-n-1}|c|^{2}
$$


Then

$$
K_{D}\left(z, \phi^{-1}(0)\right) \equiv|c|^{2}, \quad z \in D .
$$

On the other hands, if $K_{D}(z, a) \equiv|c|^{2}$ for $z \in D$, since $D$ is biholomorphically equivalent to the unit ball, there is a biholomorphic map $\psi: D \rightarrow B_{n}$ so that $\psi(a)=0$. Thus

$$
K_{D}(z, a)=\left(1-\langle\psi(z), \psi(a))^{-n-1} \operatorname{det} \psi^{\prime}(z) \operatorname{det} \psi^{\prime}(a)=\operatorname{det} \psi^{\prime}(z) \overline{\operatorname{det} \psi^{\prime}(a)}\right.
$$

Thus

$$
\operatorname{det} \psi^{\prime}(z)=\frac{|c|^{2}}{\overline{\operatorname{det} \psi^{\prime}(a)}}, \quad z \in D .
$$

Therefore, the proof of the proposition is complete. $\quad$,

\section{References.}

[1] M. S. Baouendi, P. Ebenfelt, L. P. Rothschild, Real submanifolds in complex space and their mappings. Princeton Mathematical Series, 47. Princeton University Press, Princeton, NJ, 1999.

[2] E. Bedford, Survey of Pluri-Potential Theory, Several Complex Variables: Proceedings of the Mittag-Leffler Institute, 1987-1988, John E. Fornaess, ed., Princeton University Press, Princeton, 1993, 11-65.

[3] D. Burns, Curvatures of Monge-Ampère foliations and parabolic manifolds, Ann. Math. 115 (1982), 349-373.

[4] L. Caffarelli, J. J. Kohn, L. Nirenberg and J. Spruck, The Dirichlet problem for nonlinear second-order elliptic equations, II: complex MongeAmpère, and uniformly elliptic equations, Comm. on Pure and Appl. Math. (1985), 209-252.

[5] S. Y. Cheng and S. T. Yau, On the existence of a complex Kähler metric on non-compact complex manifolds and the regularity of Fefferman's equation, Comm. Pure Appl. Math. 33 (1980) 507-544.

[6] S. S. Chern and S. Ji, On the Riemann mapping theorem, Ann. of Math., 144(1996), 421-439.

[7] C. Fefferman, The Bergman kernel and biholomorphic mapping of pseudoconvex domains, Invent. Math. 65(1974), 1-65.

[8] C. Fefferman, Monge-Ampère equations, the Bergman kernel, and geometry of pseudoconvex domains, Ann. of Math. 103(1976), 395-416. 
[9] S. Fu, B. Wong, On strictly pseudoconvex domains with Khler-Einstein Bergman metrics, Math. Res. Lett. 4 (1997), no. 5, 697-703.

[10] B. Guan, The Dirichlet problem for complex Monge-Ampère equations and Regularity of the Green's function, Comm. in Analysis and Geometry, 6(1998), 687-703.

[11] B. Guan and P. Guan, Convex Hypersurfaces of Prescribed Curvature, Ann. of Math., 156(2002), 655-674.

[12] P. Guan, Extremal Function associated to Intrinsin Norm, Ann. of Math., 156(2002), 197-211.

[13] P. Guan, N. Trudinger and X-J. Wang, Bounary regularity for degenerate Monge-Ampère equations, Acta Math., 182(1999), 87-104.

[14] Xiaojun Huang, On the rigidity of proper holomorphic maps between balls, JDG, 51(1999), 13-33.

[15] Xiaojun Huang, On some problems in several complex variables and CR geometry. First International Congress of Chinese Mathematicians (Beijing, 1998), 383-396,

[16] Xiaojun Huang and Shanyu Ji, Mapping $\mathbf{B}^{n}$ into $\mathbf{B}^{2 n-1}$, Invent. Math. 145 (2001), no. 2, 219-250.

[17] S. G. Krantz, Function Theory of Several Complex Variables, 2nd Edition, Wadsworth \& Brooks/Cole, Mathematics Series, 1992.

[18] S. G. Krantz aand S.-Y. Li, On the Existence of Smooth Plurisubharmonic Solutions for Certain Degenerate Monge-Ampère Equations, Complex variables, 41(2000), 207-219.

[19] John M. Lee, Pseudo-Einstein structures on CR manifolds, Amer. J. Math. 110 (1988), no. 1, 157-178.

[20] John M. Lee, The Fefferman metric and pseudo-Hermitian invariants, Trans. Amer. Math. Soc. 296 (1986), no. 1, 411-429.

[21] J. Lee and Melrose, Boundary behavior of the complex Monge-Ampère equation, Acta Math. (1982), 159-192.

[22] L. Lempert, Solving the degenerate Monge-Ampère equation with one concentrated singularity, Math. Ann. 263 (1983), 515-532. 
[23] S.-Y. Li, On the existence and regularity of Dirichlet problem for complex Monge-Ampère equations on weakly pseudoconvex domains, Calculus of Variations \&s PDEs, 20 (2004), 119-132.

[24] S.-Y. Li, The Neumann Problem for Complex Monge-Ampere equation, Indiana University J. of Mathematics, 44(1995), 1099-1122.

[25] S.-Y. Li, On the Dirichlet Problem for Symmetric Function Equations of Eigenvalues of Complex Hessian, Asian J. Math., 8(2004), 87-106.

[26] S-Y. Li and L. Ni, On holomorphicity of proper harmonic maps between balls with Bergman metrics, Math. Ann., 316(2000), 333-354.

[27] N. Mok and S. T. Yau, Completeness of the Khler-Einstein metric on bounded domains and the characterization of domains of holomorphy by curvature conditions. The mathematical heritage of Henri Poincar Part 1 (Bloomington, Ind., 1980), 41-59, Proc. Sympos. Pure Math., 39, Amer. Math. Soc., Providence, RI, 1983.

[28] Wilhelm Stoll, The characterization of strictly parabolic manifolds, Ann. Scuola Norm. Sup. Pisa Cl. Sci. (4) 7 (1980), no. 1, 87-154.

[29] B. Wong, Characterizations of the balls in $\mathbb{C}^{n}$ by its automorphism group, Invent. Math. 41(1977), 253-257.

[30] S. T. Yau, On the Ricci curvature of a compact Kähler maniford and the complex Monge-Ampère equation, Comm. Pure Appl. Math. 31 (1978), $339-411$.

School of Mathematics and Computer Science,

Fujian Normal University, Fujian, China

Current Address:

Department of Mathematics, University of California, IRVINE, CA 92697-3875, USA

sli@math.uci.edu

RECEIVED APRIL 13, 2004. 